\title{
La producción científica de una unidad de investigación universitaria. Una propuesta metodológica
}

\author{
Bravo Morales, Gaudy Claret*
}

\section{Resumen}

El artículo presenta una metodología para la caracterización de la producción científica de una unidad de investigación universitaria. Se registran diferentes tipos de indicadores referentes a los insumos para la investigación o indicadores de entrada y a los productos derivados de la misma o indicadores de salida, como parte de la construcción de la memoria científico-tecnológica y toma de decisiones de una organización universitaria destinada a la investigación. La metodología se aplica a un estudio de caso: Instituto de Investigaciones de la Facultad de Arquitectura y Diseño (IFAD) de La Universidad del Zulia - LUZ (Maracaibo-Venezuela), en donde la producción analizada proviene de la ejecución de proyectos de investigación financiados por el Consejo de Desarrollo Científico y Humanístico de LUZ (CONDES) entre 1987 a 1999. Las conclusiones y recomendaciones se orientan al establecimiento de correlaciones entre los indicadores registrados y las acciones gerenciales a seguir por la organización de investigación.

Palabras clave: Producción científica, unidades de investigación, investigación universitaria, evaluación científica, proyectos de investigación.

\section{Scientific Production in a University Research Unit. A Methodological Proposal}

\section{Abstract}

This article presents a methodology for the characterization of scientific production in a university research unit. Different types of indicators are registered referring to research inputs or

\author{
Recibido: 02-02-06 . Aceptado: 02-07-03
}

Arquitecta egresada de La Universidad del Zulia - LUZ (1988). MSc. en Planificación y Gerencia de Ciencia y Tecnología egresada de LUZ (2001). Miembro del personal docente y de investigación de LUZ, Instituto de Investigaciones de la Facultad de Arquitectura y Diseño (IFAD). Telefax: (58-261) 7598503, Telf: (58-261) 7598628, Apdo. Postal 4011-A-526, Maracaibo, Venezuela (e-mail: gbravo@luz.ve) 
entry indicators and the products derived from the same (output) in order to construct a scientific-technology memory and decision-making process for a university organization destined to do research. The methodology applied was a case study: the Research Institute (IFAD) at the Faculty of Architecture and Design (IFAD) at the University of Zulia, (LUZ) Maracaibo, Venezuela, where the research production analyzed comes from the research projects executed and financed by the Scientific and Humanistic Research Council (CONDES) between 1987 and 1999. The conclusions and recommendation are oriented towards establishing correlations between indicators registered and management actions to be followed by the research organization.

Key words: Scientific production, research units, university research, scientific evaluation, research projects.

\section{Introducción}

Los indicadores de la actividad y/o producción científica, entendidos como el conjunto de datos que responden a preguntas concretas sobre las condiciones 0 cambios de la estructura interna y relaciones externas del trabajo científico, se han utilizado en respuesta a las crecientes presiones económicas mundiales como una forma de evaluar la distribución de las asignaciones presupuestarias en materia científica y tecnológica.

Los criterios de distribución y asignación presupuestaria tradicionalmente se designan desde el interior de cada disciplina y se completan con la evaluación de los "pares". Mientras que, la producción científica de las instituciones e investigadores es casi exclusivamente evaluada a través de los datos bibliométricos del Science Citation Index, con el argumento de que los mismos ofrecen unos resultados más visible internacionalmente (CONICIT, 1995). Con la selectividad en la distribución del presupuesto para la investigación, como resultado de las transformaciones ocurridas en el sector científico y las disminuciones presupuestarias, se buscan nuevas formas o métodos de evaluación no solamente a través de indicadores económicos, sino también sociales. Para López (1998), es importante distinguir entre una evaluación de la estructura de la investigación y una evaluación de los resultados de la investigación. En este último caso, Licea de Arenas (1993) plantea que se deben considerar dos tipos básicos de indicadores: primero, los indicadores de entrada o insumos para la investigación, tales como, los recursos, los investigadores, el personal de apoyo, los equipos y las instalaciones y segundo, los indicadores de salida, tales como los productos de la actividad científica, las actividades educativas y las innovaciones tecnológicas.

En el contexto nacional venezolano, el entonces Consejo Nacional de Investigaciones Científicas y Tecnológicas de Venezuela (CONICIT) ahora Fondo Nacional de Ciencia y Tecnología (FONACIT), reporta anualmente varios indicadores nacionales de la capacidad de investigación en Ciencia y Tecnología (C y $\mathrm{T}$ ) que reflejan cuantitativamente más no cualitativamente, los recursos otorgados al desarrollo científico y a la actividad de investigación dentro de las universidades $u$ otras entidades públicas. Algunos 
de estos indicadores, registran el número de investigadores, el número de centros de I y D, la inversión del Estado en C y T, los cursos de postgrado, el número de becas otorgadas (formación de recursos), el número de proyectos de investigación financiados, entre otros (CONICIT, 1995).

En el ámbito de una organización, autores como Ferrer de Esis (1997), Peña y Barrios (1994) y Paredes (1993), señalan que los productos de la actividad científica y tecnológica deben orientarse hacia la generación de innovaciones, desarrollos tecnológicos y prestación de servicios tecnológicos. Al mismo tiempo, estos autores señalan que la atención a pasantes, la preparación de investigadores, la participación en cursos de postgrado y la actualización de los recursos humanos también son productos obtenidos de la relación entre la actividad de investigación con la docencia y la capacitación. Para De Vries (1998), tanto los productos de la actividad de investigación y desarrollo (I y D) como los indicadores para medir o evaluar la producción de la investigación científica, dependen del contexto socio-económico y científico-tecnológico, del enfoque teórico y de los objetivos del estudio o evaluación

Con esta información sobre indicadores asociados con la actividad y producción científica-tecnológica, se indagan otras referencias sobre unidades de investigación científica universitaria; e incluso, aplicaciones de propuestas metodológicas para registrar, evaluar y caracterizar la actividad y producción científica de dichas unidades. Al respecto, para el momento de la elaboración del estudio (1998-2000) no se encontró información impresa y registrada en las bibliotecas de
LUZ (Facultad Experimental de Ciencias, Facultad de Humanidades y Educación, Facultad de Ingeniería, Facultad de Arquitectura, Sistema de Servicios Bibliotecarios e Información - SERBILUZ, Centro de Documentación, Información y Archivo - CEDIA y CONDES) y del antiguo CONICIT, ni en las bases de datos de diferentes buscadores de Internet para referencias nacionales e internacionales. Los estudios de casos encontrados en el contexto nacional, tales como los publicados por Licha (1993) y realizados en diferentes unidades de la Universidad Central de Venezuela y los realizados por Castro (1994), González (1995), de Azuaje (1987) y Figueroa (1995) en unidades de LUZ y empresas locales, se fundamentan básic amente en la identificación de factores que limitan la actividad y producción científica universitaria, en el análisis y propuesta de mecanismos de vinculación entre la institución de investigación y el sector productivo y en el diseño de propuestas gerenciales concretas para las actividades de I y D. Estos tres tipos de estudios consideran aspectos que caracterizan la organización y que obstaculizan la actividad de investigación.

La limitación de información, determina la necesidad de definir y precisar una metodología para caracterizar la producción científica de una unidad de investigación universitaria derivada fundamentalmente de la ejecución de proyectos de investigación y considerando que es la forma primaria de gestionar (formalmente) la actividad científica. Tal información, es parte esencial para establecer un conjunto de acciones gerenciales viables con el propósito de potenciar la producción científica en los procesos estratégicos de 
planificación, control y evaluación de la actividad científica y para facilitar el proceso de construcción de la memoria científico-tecnológica de una organización de investigación universitaria, orientada a la solución de los problemas del entorno socio-económico y como una forma de contribuir con el desarrollo nacional competitivo, a través del estímulo y desarrollo de innovaciones científico-tecnológicas.

Algunos aspectos de la metodología y de los resultados derivados de la aplicación a un caso de estudio, se presentan en este artículo.

\section{Metodología}

Para cumplir con el propósito central formulado en este trabajo, se propone una metodología que combina elementos de la investigación exploratoria y descriptiva de carácter retrospectivo al fundamentar la producción científica de la unidad de investigación en proyectos de investigación ejecutados y/o culminados durante un período de tiempo lo suficientemente amplio para obtener datos registrados en documentos escritos y conclusiones históricamente significativas. La metodología aborda aspectos cuantitativos y algunos cualitativos no tratados en este artículo.

\subsection{Fuentes de datos}

La recolección de los datos, se realiza a partir de los expedientes de cada uno de los proyectos de investigación archivados en los entes financiadores (Consejos de Desarrollo Científicos, Humanísticos y Tecnológicos o CDCHT, Unidad de Investigación, otros) y se refie- re básicamente a dos tipos de indicadores (López, 1998): los indicadores de entrada o insumos para la investigación y los indicadores de salida, tales como los productos de la actividad científica y las actividades educativas desarrolladas con o durante la ejecución del proyecto.

Los indicadores a registrar se limitan a las siguientes variables: los montos aprobados por el ente universitario financiador (CDCHT) y ejecutados por cada uno de los entes que financian los proyectos de investigación de la unidad de investigación universitaria, los tiempos administrativos de aprobación y ejecución de los proyectos de investigación de la unidad de investigación, datos personales relacionados con el personal docente-investigador de los proyectos de investigación de la unidad, datos relacionados con el personal contratado para laborar en los proyectos de investigación de la unidad u organización, y por último, los datos relacionados con los equipos adquiridos por los proyectos de investigación y financiados por el CDCHT.

Los indicadores de salida se refieren a los productos generados por cada uno de los proyectos de investigación. En este sentido, se registran y analizan productos directos y productos derivados 0 conexos.

Los productos directos obtenidos de los proyectos de investigación, los cuales dependen de la naturaleza y del proceso de investigación seguido por cada proyecto, son entendidos como aquellos que tienen su fundamento en el planteamiento y cumplimiento de los objetivos básicos de la investigación, así como del proceso mismo de investigación. Se puede considerar como produc- 
tos directos a: las evaluaciones y análisis (procesos y resultados), los métodos o modelos de evaluación y análisis, la generación de base de datos, los registros documentales, las caracterizaciones, los diseños, construcción y evaluación de modelos conceptuales, herramientas, plataformas experimentales, propuestas, entre otros.

Los productos derivados o conexos obtenidos con o a partir del desarrollo de los proyectos de investigación de la unidad, son aquellos productos relacionados con: por un lado, la divulgación, difusión y/o promoción del conocimiento y de los productos directos obtenidos en los diferentes proyectos (ver Cuadro 1), tales como: el material bibliográfico producido o las publicaciones generadas, la participación y organización de eventos científicos, y por último, otros medios o contactos para la difusión del conocimiento; y por otro lado, con las actividades educativas registradas y directamente vinculadas con los proyectos de investigación.

\subsection{Procedimiento para la recolección de información}

La información documental (variables e ítems), se registra en unas planillas previamente diseñadas y elaboradas en formato impreso y digital mediante el diseño de una base de datos relacional para su posterior procesamiento y consulta. La revisión documental implica la lectura detallada y cuidadosa de cada documento producido por la investigación.

El procedimiento para determinar los insumos es el siguiente:

1. Estimación de los montos aprobados y ejecutados por proyecto de investi- gación. En este rubro, se procesan los montos aprobados por proyecto y por partida financiada por el ente financiador, la ejecución presupuestaria del monto otorgado por el ente universitario financiador (CDCHT) por rubros financiados y totales por proyecto (monto total), los montos aprobados y ejecutados en valor constante de la moneda local para una misma base de proyección (mes y año) con el propósito de comparar los costos bajo un mismo valor de la moneda y observar el efecto de la devaluación e inflación durante el período de estudio seleccionado, los montos aprobados y ejecutados en dólares estadounidenses (US\$) en valor corriente y constante para referir los costos a una moneda internacional, el monto equivalente por la participación del personal docente-investigador en cada uno de los proyectos de investigación (en moneda local y en US\$ a valor corriente y constante), y por último, el monto global ejecutado por cada proyecto de investigación (en moneda local y en US\$ a valor corriente y constante).

2. Los tiempos administrativos (parciales y totales) para la aprobación de la solicitud de financiamiento ante el ente financiador. En este sentido, se estima la duración total del trámite administrativo considerando el inicio del primer trámite hasta el inicio del proyecto. Igualmente, se estima la duración promedio de los proyectos considerando la fecha de inicio y la fecha de culminación de los mismos, con sus límites máximos y mínimos de duración.

3. Las características del personal docente-investigación, del personal 


\section{Cuadro 1 \\ Producción Científica \\ Indicadores de los productos indirectos}

\begin{tabular}{|c|c|c|}
\hline Productos & Categorías & Datos registrados \\
\hline Libros & $\begin{array}{l}\text { - Libros } \\
\text { - Capítulos o Artículos de Libros } \\
\text { - Sin información } \\
\text { - Sin publicación }\end{array}$ & $\begin{array}{l}\text { - Identificación del } \\
\text { proyecto (IP) } \\
\text { - Título del libro o capítu- } \\
\text { lo } \\
\text { - Autores } \\
\text { - Año o edición } \\
\text { - Editorial } \\
\text { - No. de ejemplares } \\
\end{array}$ \\
\hline $\begin{array}{l}\text { Artículos en revista } \\
\text { científica }\end{array}$ & $\begin{array}{l}\text { - Arbitrado (evaluados y aprobados por árbitros, publicados o no) } \\
\text { - En arbitraje (recibidos y en fase de arbitraje) } \\
\text { - Recibido para publicar (recibido sin iniciar proceso de arbitraje) } \\
\text { - No arbitrado (aceptados o publicados sin arbitraje). } \\
\text { - Sin artículos } \\
\text { - Sin información (documentada) } \\
\text { - Escala de publicación: nacional e internacional }\end{array}$ & $\begin{array}{l}\text { - IP } \\
\text { - Título del artículo } \\
\text { - Añores } \\
\text { - Nombre revista } \\
\text { - Número y volumen }\end{array}$ \\
\hline $\begin{array}{l}\text { Artículos en memo- } \\
\text { rias de eventos } \\
\text { científicos }\end{array}$ & $\begin{array}{l}\text { - Arbitrado } \\
\text { - No arbitrado } \\
\text { - Eecibido para publicar } \\
\text { - Sin artículo } \\
\text { - Sin información }\end{array}$ & $\begin{array}{l}\text { - } \text { IP } \\
\text { - } \text { Aútulo del artículo } \\
\text { - Año } \\
\text { - Nombre del Evento } \\
\text { - Lugar de realización }\end{array}$ \\
\hline $\begin{array}{l}\text { Monografías y/o in- } \\
\text { formes científicos }\end{array}$ & - No publicaron & $\begin{array}{l}\text { - } \text { IP } \\
\text { - } \text { Autulo } \\
\text { - Añores } \\
\text { - Año }\end{array}$ \\
\hline Otras publicaciones & $\begin{array}{l}\text { - Con publicaciones: folletos o trípticos, reseñas en periódicos de cir- } \\
\text { culación regional, nacional e internacional, reseñas en boletines inter- } \\
\text { nos y externos, otros medios (revistas no científicas, afiches, concur- } \\
\text { sos, otros) } \\
\text { - Sin publicaciones (que no tiene "otras publicaciones") } \\
\text { - Sin información }\end{array}$ & $\begin{array}{l}\text { - } \text { IP } \\
\text { - Nombre de la pu- } \\
\text { blicación } \\
\text { - Año } \\
\text { - Lugar de realización } \\
\text { - Título del artículo } \\
\text { - Autores }\end{array}$ \\
\hline Asistencia a eventos & $\begin{array}{l}\text { - Modalidad de presentación: oral o afiche } \\
\text { - Escala del evento: nacional o internacional } \\
\text { - Tipo de evento: Congreso, conferencias, simposio, seminario, taller u } \\
\text { otros (foros, jornadas, encuentro, charlas, entre otros) } \\
\text { - Sin asistencia } \\
\text { - Sin información }\end{array}$ & $\begin{array}{l}\text { - IP } \\
\text { - Nombre del evento } \\
\text { - Lugar de realización } \\
\text { - Paño } \\
\end{array}$ \\
\hline $\begin{array}{l}\text { Organización de } \\
\text { eventos }\end{array}$ & $\begin{array}{l}\text { - Con organización } \\
\text { - No se organizaron } \\
\text { - Sin información }\end{array}$ & $\begin{array}{l}\text { - IP } \\
\text { - Nombre del evento } \\
\text { - Lugar de realización } \\
\text { - Año } \\
\text { - Patrocinadores }\end{array}$ \\
\hline $\begin{array}{l}\text { Otros medios o con- } \\
\text { tactos de difusión }\end{array}$ & $\begin{array}{l}\text { - Tipo de Comunicación: personal e institucional establecida por los } \\
\text { investigadores durante la ejecución del proyecto } \\
\text { - Tipo de organismo: públicos o empresas privadas. } \\
\text { - Escala del contacto: nacionales o internacionales } \\
\text { - Sin contacto } \\
\text { - Sin información }\end{array}$ & $\begin{array}{l}\text { - } \text { IP } \\
\text { - Nombre de la persona } \\
\text { o institución } \\
\text { - Lugar del co ntacto }\end{array}$ \\
\hline $\begin{array}{l}\text { Actividades educati- } \\
\text { vas registradas du- } \\
\text { rante el proyecto }\end{array}$ & $\begin{array}{l}\text { - Cursos, exposiciones y charlas realizadas por algún miembro del equi- } \\
\text { po de investigación: dentro del recinto universitario y fuera del recinto } \\
\text { - Asesoramiento a estudiantes de pre y postgrados de la universidad y } \\
\text { de otras instituciones: durante pasantías, tesis de grado, escolaridad } \\
\text { - Asesoramiento a otros profesores de la universidad y de otras institu- } \\
\text { ciones } \\
\text { - Asesoramiento a instituciones o empresas. }\end{array}$ & $\begin{array}{ll}\text { - } & \text { IP } \\
\text { - Nombre de la actividad } \\
\text { - Lugar } \\
\text { - } \text { Fecha } \\
\text { - Expositor/autor }\end{array}$ \\
\hline
\end{tabular}

Fuente: Bravo, 2000 
contratado y de los equipos adquiridos en los diferentes proyectos. Se estiman las características predominantes del personal y de los equipos determinando la frecuencia.

4. Las áreas de conocimiento donde se adscriben los proyectos de investigación. Se determinan las áreas más frecuentemente registradas e investigadas durante el período de estudio.

Mientras que, para registrar y listar los productos directos reportados por los investigadores en los proyectos de investigación, se hacen lecturas de los diferentes informes científicos por proyectos. Estos se organizan y agrupan en productos comunes por proyectos y en un listado general donde se elabora una categorización general y sub-categorización de productos. Posteriormente, se cuantifica y determina el porcentaje de representatividad de las sub-categorías respecto a las categorías y de las categorías generales con respecto al total de categorías obtenidas. Los resultados se representan gráficamente.

Para el registro de los productos derivados o conexos, se propone la revisión cuidadosa de los datos reportados en los informes finales de los proyectos de investigación. Estos datos, se deben transcribir en la planilla de registro y en la base de datos. La información se procesa para realizar una distribución numérica y porcentual por ítems.

\subsection{Procedimiento para determinar la producción científica de la unidad de investigación}

Para determinar la producción científica de una unidad de investigación universitaria (Bravo, 2001), se establece una doble relación porcentual y comparativa entre: los montos globales ejecutados por cada uno de los proyectos de investigación estudiados y el total ejecutado por el conjunto de proyectos en un período determinado y entre los productos totales generados por cada proyecto de investigación respecto al total de productos generados en el conjunto estudiado en el mismo período de análisis. En este sentido, es necesario totalizar los productos obtenidos (directos+indirectos) por proyecto y por el conjunto de proyectos.

\section{Aplicación de la metodología en un caso de estudio}

La metodología propuesta se aplica a un caso concreto e intencional de estudio para caracterizar su producción científica. En el caso del Instituto de investigaciones - IFAD, de la actual Facultad de Arquitectura y Diseño (FAD) de la Universidad del Zulia (LUZ) ubicado en la ciudad de Maracaibo-Venezuela. Este instituto, se inicia aproximadamente en 1978, con el propósito de "ampliar el campo de interés de la labor de investigación" a otros problemas de investigación propios de la entonces Escuela de Arquitectura (LUZ, 1978; CIUR, 1979; Bravo, 1996). Desde ese momento, los problemas se asociaron a tres áreas de interés y trabajo, a saber: Acondicionamiento Ambiental, Sistemas Constructivos (hoy Hábitat y Vivienda) y Sistemas de Información y Documentación. Entre 1995 y 1996, se incluye el área de Patrimonio y Turismo para dar cabida a las investigaciones históricas en relación con el patri- 
monio arquitectónico y a una disciplina impartida hace tiempo en el postgrado de la Facultad (turismo). Actualmente, se mantienen estas áreas de investigación.

Ahora bien, en el caso particular del IFAD, no se conocía la situación formal e histórica de su producción en tanto que, no se encontraron registros en el IFAD sobre investigaciones previas relacionadas con el tema. Por tal razón, y considerando la ausencia y deficiencia de información académica y administrativa de los proyectos no financiados por el CONDES (CDCHT de LUZ) y de otras actividades ejecutadas consecutivamente en el IFAD, se seleccionaron los proyectos aprobados por el CONDES durante el período 1986-1996 y ejecutados en el período 1987-1999. Esta selección está con- formada por 27 proyectos aprobados entre 1986-1996, de los cuales 26 se ejecutaron y 23 culminaron durante ese período (1987-1999).

\section{Discusión de resultados obtenidos en el caso de estudio}

\subsection{Montos aprobados por el CONDES para los proyectos del IFAD}

El comportamiento anual de los montos aprobados para los proyectos de investigación durante el período de estudio, calculados en la moneda local (Bolívares) a valor corriente y a valor constante en diciembre de 1999 (Banco Central

\section{Gráfico 1 \\ Comparación de los montos aprobados para los proyectos de investigación IFAD-CONDES (1986-1996) en bolívares corrientes y constantes}

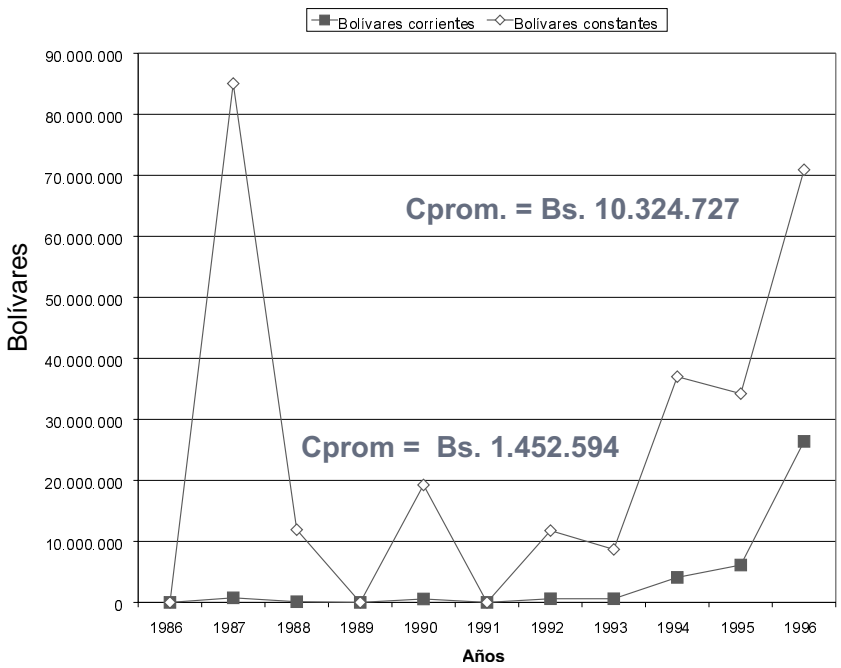

Fuente: Memorias y Cuentas CONDES desde1986 a 1996 y estimación propia (Bravo, 2000). 
de Venezuela, 2000), se observa en el Gráfico 1. La sustancial diferencia evidenciada entre ambos valores de la moneda, se debe fundamentalmente al efecto de la devaluación monetaria e inflación ocurrida en el país durante ese período. Se aprecia además, los años donde hubo mayores montos aprobado por concepto de proyectos de investigación (años '87, '96 y '94), los cuales coinciden con el mayor número de proyectos aprobados por año $(6,8$ y 3 proyectos respectivamente), y los años donde no hubo aprobación de proyectos IFAD-CONDES ( ' 86 , ' 89 y ' 91 ). Además, se reportan los costos promedios (Cprom) aprobados de los proyectos por año y durante el período de estudio, estimados a valor corriente y a valor constante. En este sentido, se obtiene que el Cprom aprobado en valor constante es aproximadamente 7 veces mayor al costo en valor corriente.

En el Gráfico 2, se muestran los mismos montos aprobados para los proyectos IFAD-CONDES pero estimados en dólares estadounidenses (US\$) a valor corriente (Kauffman y Asociados, 1999) y constante del mismo mes y año de referencia que el caso anterior (U.S. Department of Labor Bureau of Labor Statistics, 2000). Se observa, que el comportamiento anual es similar entre ambos valores y eso es justamente debido a las escasas variaciones en el valor de esa moneda (y menor inflación) respecto a la moneda local. Durante los once años de

\section{Gráfico 2 \\ Comparación de los montos aprobados para los proyectos de investigación IFAD-CONDES (1986-1996) en dólares USA corrientes y constantes}

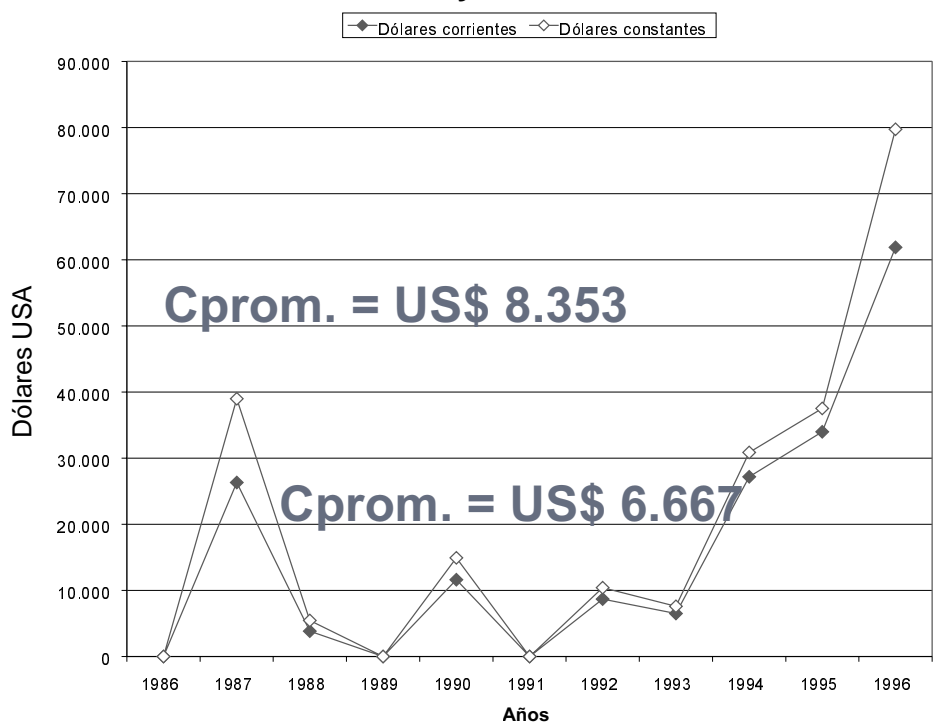

Fuente: Estimación propia (Bravo, 2000). 
estudio, se estima que el Cprom aprobado de un proyecto IFAD-CONDES en US $\$$ a valor constante es 1,25 veces mayor que el valor de la moneda a valor corriente.

\subsection{Montos totales ejecutados por los proyectos IFAD-CONDES}

Los montos totales ejecutados por proyecto en bolívares corrientes y constantes y los costos promedios (Cprom) a valor constante ejecutados por proyecto durante el período de estudio, se presentan en el Gráfico 3. En la misma, se observan los proyectos que ejecutaron más dinero.

En bolívares constantes, los montos ejecutados por proyecto ascendieron a un poco más de 15 millones de bolívares, siendo el Cprom de 6 millones aproximadamente de 22 proyectos ejecutado y culminados para diciembre de 1999 (un proyecto no ejecutó financiamiento $\mathrm{CON}$ DES). Por otra parte, al relacionar los montos totales aprobados y los montos totales ejecutados por los proyectos IFAD-CONDES, se obtiene que en tres (3) años los montos ejecutados fueron superiores a los aprobados inicialmente por el CONDES con un incremento promedio de $156 \%$. Estos incrementos, fueron absorbidos por el CONDES durante la ejecución de los proyectos y en parte, fueron consecuencia del efecto de la inflación y devaluación de la moneda nacional. El promedio de ejecución en relación con lo aprobado asciende al $81 \%$.

\section{Gráfico 3}

Comparación de los montos totales ejecutados por los proyectos de investigación IFAD-CONDES (1986-1996) en bolívares corrientes y constantes

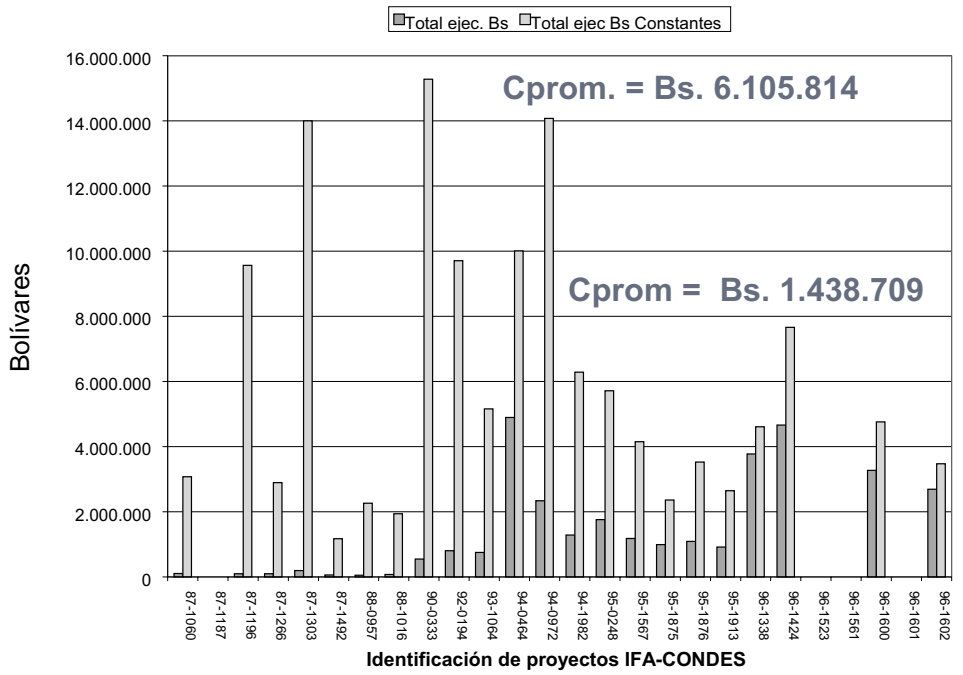

Fuente: Memorias y Cuentas CONDES desde1986 a1996 y estimación propia (Bravo, 2000). 


\section{Gráfico 4}

Comparación de los montos totales ejecutados por los proyectos de investigación IFAD-CONDES (1986-1996) en dólares USA corrientes y constantes

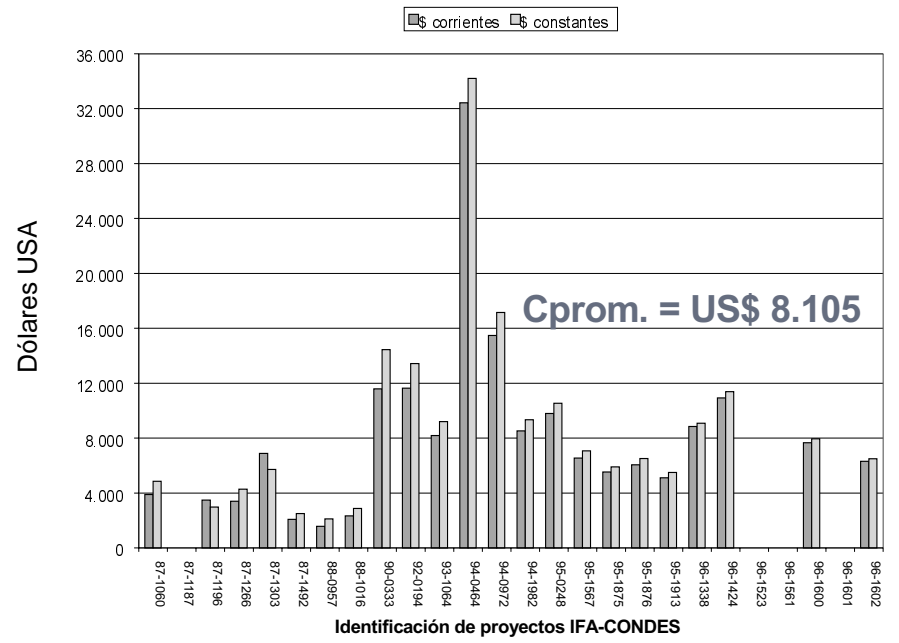

Fuente: Memorias y Cuentas CONDES desde1986 a1996 y estimación propia.

Los mismos montos totales y Cprom ejecutados referidos anteriormente, se presentan en el Gráfico 4, pero en US\$ a valor corriente y constante. En este caso, los montos ejecutados por proyecto ascienden a 34 mil US\$ a valor constante aproximadamente y el Cprom por proyecto asciende a más de 8 mil US\$ a valor constante.

\subsection{Relación montos ejecutados por partidas con montos totales ejecutados en los proyectos IFAD-CONDES}

La distribución de los montos ejecutados por partidas financiadas por el CONDES se muestra en el Gráfico 5 y en la Tabla 1, en donde las partidas de "personal" y "equipo", ejecutaron la mayor parte del monto aprobado y absorbieron el $83 \%$ del monto ejecutado en relación con los otros rubros financiados.

\subsection{Montos ejecutados por el personal docente-investigador en los proyectos IFAD-CONDES}

Los montos en bolívares corrientes y constantes ejecutados por el personal docente y de investigación LUZ, que laboraron en los proyectos IFAD-CONDES y el Cprom en valor constante, se muestra en el Gráfico 6. En la misma, se observa que los montos en bolívares constantes de 3 proyectos oscilan entre 320 a 200 millones de bolívares, siendo el Cprom de los 23 proyectos de aproximadamente 81 millones de bolívares. 


\section{Gráfico 5}

Porcentajes de representación de los montos ejecutados por partidas con relación a los montos totales ejecutados en los proyectos IFAD-CONDES organizados en años de aprobación de los proyectos

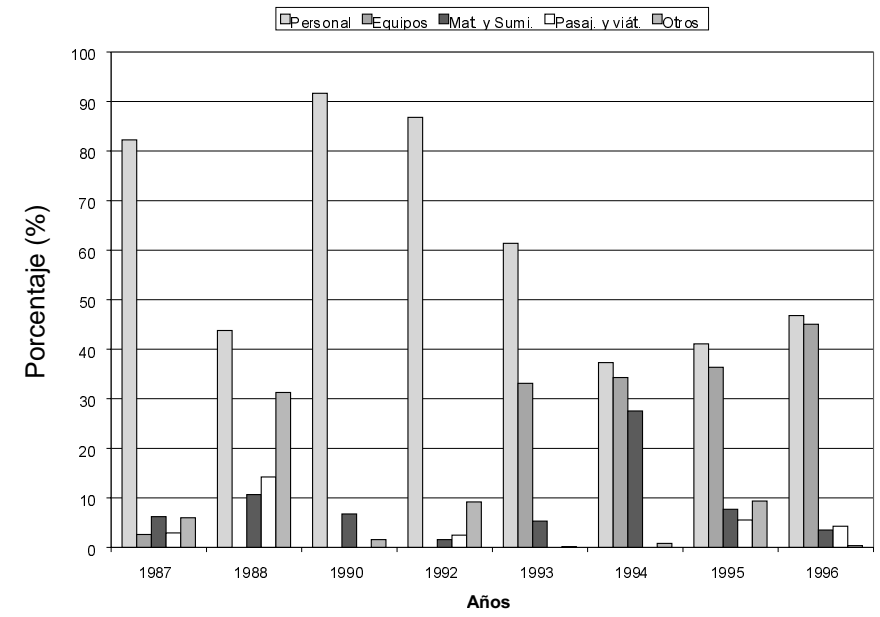

Fuente: Estimación propia (Bravo, 2000).

Tabla 1

Porcentajes de representación de los montos ejecutados por partidas con respecto a los montos ejecutados en los proyectos IFAD-CONDES

\begin{tabular}{|ccccccc|}
\hline $\begin{array}{c}\text { No. Total de } \\
\text { proyectos }\end{array}$ & Total (\%) & Personal & Equipos & $\begin{array}{c}\text { Materiales y Su } \\
\text { ministro }\end{array}$ & $\begin{array}{c}\text { Pasajes } \\
\text { y viáticos }\end{array}$ & Otros \\
\hline 22 & 100 & 45,93 & 37,39 & 10,88 & 3,16 & 2,64 \\
\hline
\end{tabular}

Fuente: Estimación propia (Bravo, 2000).

Los mismos montos pero en US\$ a valor corriente y constante se comparan en el Gráfico 7. En dólares constantes, los montos ascienden entre 500 a $290 \mathrm{mil}$ US\$ en 3 proyectos, estimándose el Cprom cerca de 122 mil US\$.

\subsection{Montos globales de los} proyectos de investigación

El monto global en esta investigación se limita a la ejecución de los montos parciales debido a la participación del personal docente y de investigación de LUZ, CONDES y co-financiamientos externos. Los otros costos no se consideran por falta de información. En este sentido, la comparación de los montos parciales y su aporte al monto global ejecutado por cada proyecto de investigación y el Cprom, se muestran en los gráficos 8 y 9 , en bolívares y dólares constantes, respectivamente. 


\section{Gráfico 6}

Montos ejecutados debido a la participación del personal docente y de investigación en los proyectos IFAD-CONDES (1986-1996), en bolívares corrientes y constantes

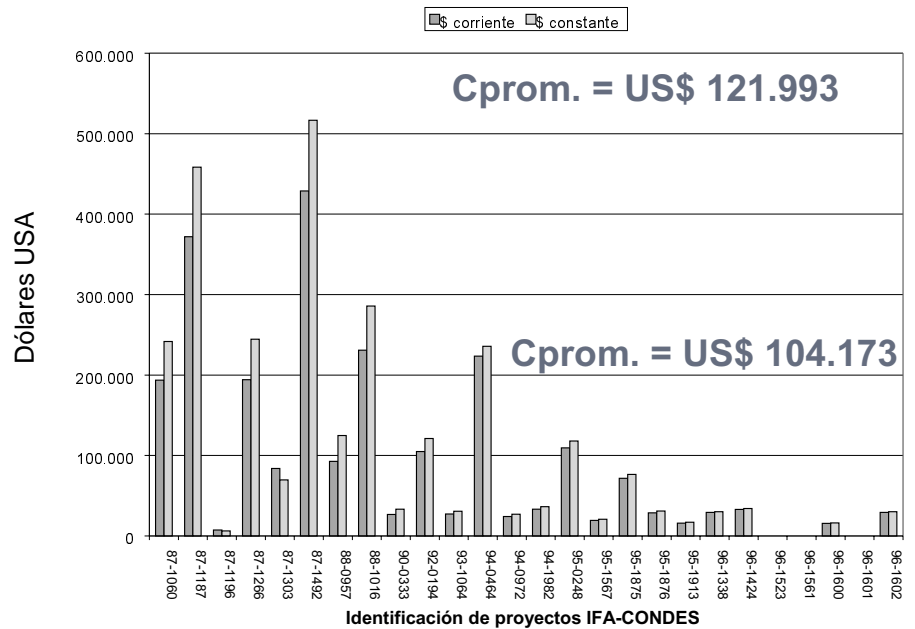

Fuente: Estimación propia (Bravo, 2000).

\section{Gráfico 7}

Montos ejecutados debido a la participación del personal docente y de investigación en los proyectos IFAD-CONDES (1986-1996), en dólares corrientes y constantes

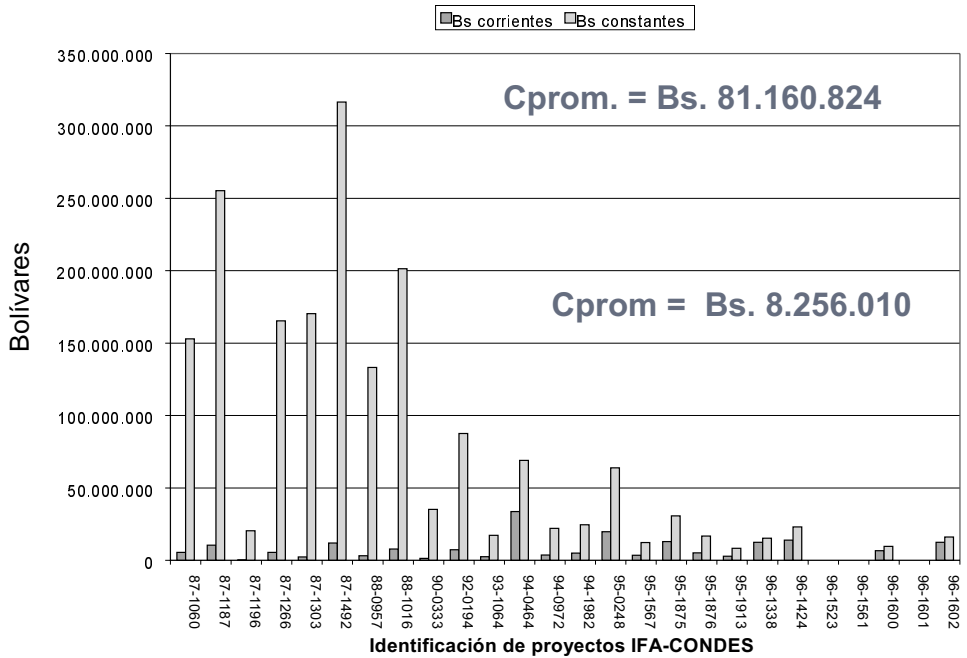

Fuente: Estimación propia (Bravo, 2000). 


\section{Gráfico 8 \\ Relación de los montos ejecutados por el personal LUZ, los co-financiamientos y el CONDES en los proyectos de investigación IFAD-CONDES, en bolívares corrientes y constantes}

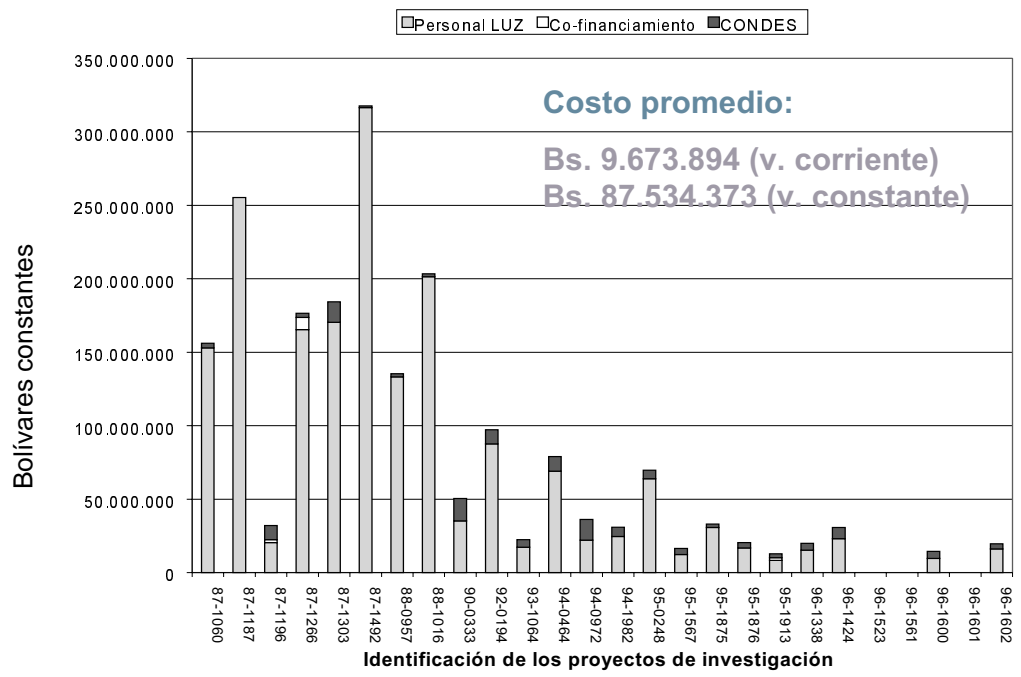

Fuente: Estimación propia (Bravo, 2000).

En el Gráfico 8, se observa que 7 proyectos que fueron aprobados entre 1987 y 1988, ejecutaron los mayores montos globales durante el lapso de estudio, es decir, con montos que sobrepasan los 100 millones de bolívares en valor constante. De estos proyectos y en valores constantes, el de mayor ejecución alcanzó la cifra de más de 317 millones de bolívares (más de 500 mil US\$) y el Cprom global estimado por proyecto sobrepasa los 87 millones de bolívares y algo más de 131 mil US $\$$ en valores constantes (ver Gráfico 9). Bajo esta estimación, el monto global ejecutado de los 23 proyectos ascendió a 2.013 millardos de bolívares a valor constante.

Durante el período de estudio, del monto global ejecutado en los proyectos
IFAD-CONDES apenas el 6,7\% corresponde a los montos aprobados y ejecutados con financiamiento del CONDES, el $92,7 \%$ al costo de la participación del personal docente-investigador de la universidad (LUZ) y el $0,6 \%$ al financiamiento recibido por entes externos (co-financiamiento).

\subsection{Tiempos administrativos de los proyectos IFAD-CONDES}

Los tiempos mínimos, máximos y promedios de cada instancia administrativa para la aprobación del financiamiento a través del CONDES y la duración promedio de los proyectos, se muestra en el Diagrama 1. Al respecto, se obtiene que el tiempo promedio que demoró la trami- 


\section{Gráfico 9}

Relación de los montos ejecutados por el personal LUZ, los co-financiamientos y el CONDES en los proyectos de investigación IFA-CONDES, en dólares corrientes y constantes

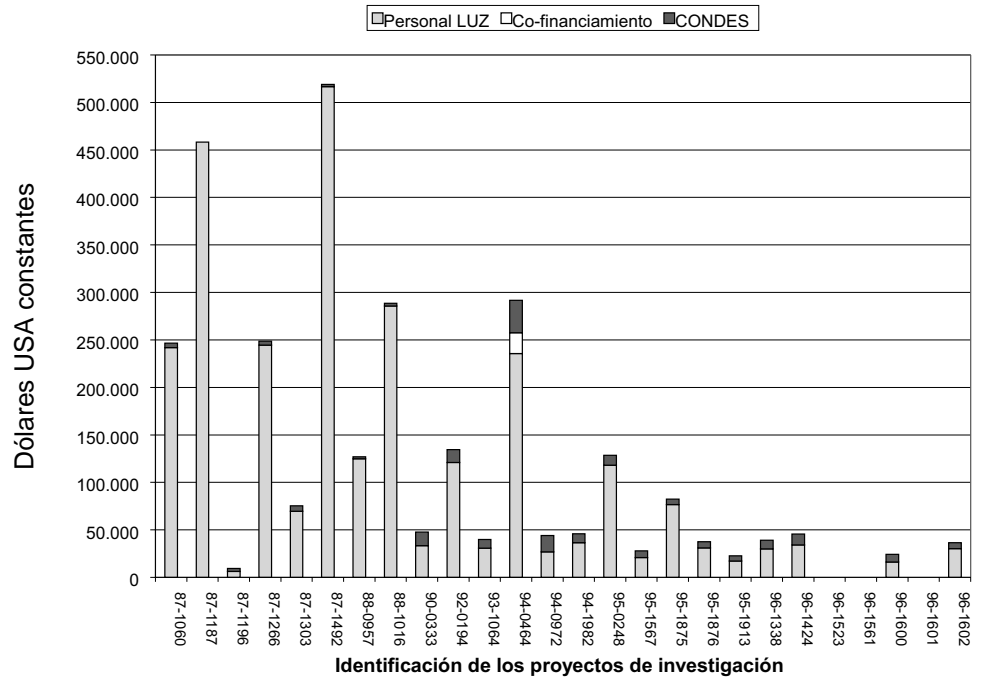

Fuente: Estimación propia (Bravo, 2000).

\section{Diagrama 1}

Tiempos administrativos para la aprobación y duración de los proyectos de investigación IFAD-CONDES

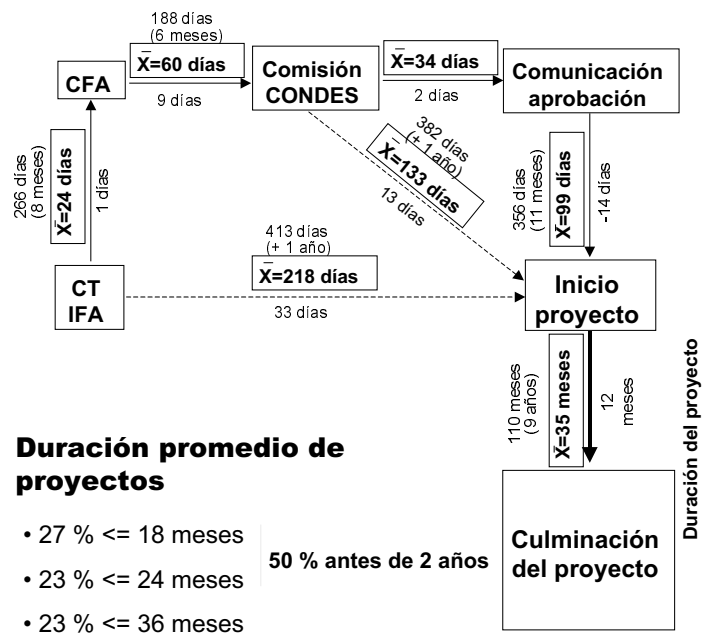

Fuente: Estimación propia (Bravo, 2000). 
tación para la aprobación de los proyectos IFAD-CONDES, fue de aproximadamente 3 meses a partir de la fecha de aprobación ante el Consejo Técnico del IFAD (CT IFAD) y la fecha de la comunicación de aprobación del CONDES. Mientras que, el tiempo promedio que demoró el proyecto de investigación en iniciar sus actividades oficialmente desde la aprobación de la Comisión CONDES fue de 4 meses y medio. Por otra parte, el promedio de duración de los proyectos ejecutados IFAD-CONDES (23 proyectos) fue de casi 3 años (35 meses). En este sentido, los proyectos de investigación con mayor tiempo de ejecución fueron los aprobados en 1987 y 1988.

\subsection{Características del personal docente-investigador, personal contratado y equipos adquiridos}

Con respecto al personal docenteinvestigador de LUZ que laboró en los diferentes proyectos de investigacióo IFADCONDES, se obtiene que un poco más de la mitad fue en calidad de co-investigador (57\%), el $82 \%$ estaba con dedicación exclusiva, su participación promedio fue de 13 horas semanales, su edad promedio fue de 36 años, con una categoría predominante de Titular y Agregado (61\%) y con un predominio de formación de cuarto nivel $(53 \%)$. Por otro lado, más de la mitad del personal contratado y pagado por el CONDES, trabajó en calidad de colaborador y un tercio en calidad de auxiliar. La formación predominante fue en arquitectura $(76 \%)$ y con una jornada laboral promedio de 22 horas a la semana (72\%).

Los equipos adquiridos a través de los proyectos IFAD-CONDES fueron prin- cipalmente computadoras, software y periféricos (84\%), las cuales se concentraron en los proyectos aprobados entre 1992 y 1996 (los proyectos anteriores no solicitaron estos equipos). El tiempo promedio de adquisición estimado de estos equipos alcanza los 12 meses ( 1 año) desde la fecha de aprobación del CON. DES hasta la fecha de la factura de compra.

\subsection{Areas de conocimiento}

Se obtuvo un listado de 19 áreas de conocimiento en 24 proyectos IFADCONDES ( 2 proyectos no tenían información), en donde "vivienda" y "diseño bioclimático y ahorro de energía" fueron las áreas o líneas de conocimiento donde se adscribieron mayormente los proyectos IFAD-CONDES. No obstante, considerando la falta de una clara definición de áreas y líneas, así como una inconsistencia recurrente en la forma como los proyectos las refieren, puede decirse que un $44 \%$ de los proyectos abordaron estudios de carácter urbano, seguidos de un $38 \%$ relacionados a la tecnología de la construcción de edificaciones.

\subsection{Productos directos generados}

Los productos directos generados por los proyectos de investigación IFADCONDES y sus frecuencias se muestran en el Cuadro 2. Los "registros documentales" (cronológicos, morfológicos, cartográficos, gráficos, cuantitativos, de experiencias o casos, de modelos y métodos, teóricos-conceptuales, planimétricos y fotográficos) ocupan el $32 \%$ de los productos totales generados (352 produc- 


\section{Cuadro 2}

\section{Clasificación de los productos directos según categorías generales. Frecuencia derivada de 26 proyectos de investigación IFAD-CONDES}

\begin{tabular}{|c|c|c|c|}
\hline No. & Categoría General & Definiciones & Frecuencia \\
\hline 1 & Registro documental & 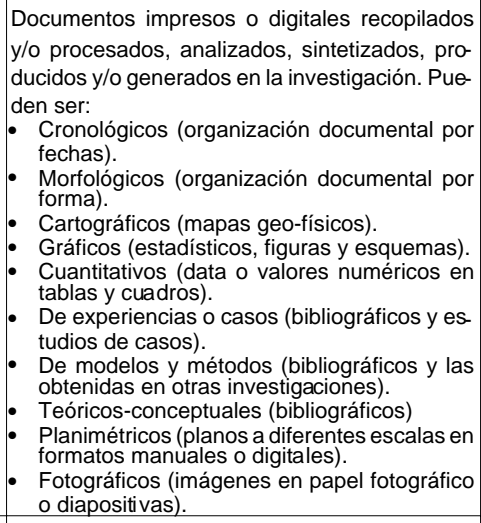 & 49 \\
\hline 2 & Evaluación y Análisis & Procesos y resultados & 33 \\
\hline 3 & Modelo-método de evaluación y análisis & $\begin{array}{l}\text { Analógicos o digitales diseñados para la evalua- } \\
\text { ción y análisis. }\end{array}$ & 28 \\
\hline 4 & Base de datos & $\begin{array}{l}\text { Referidas a los registros de datos (numéricos, } \\
\text { gráficos, fotográficos, planimétricos, cartográfi- } \\
\text { cos, sonoros) en formatos pre establecidos para } \\
\text { su almacenamiento y consultas relacionales. }\end{array}$ & 10 \\
\hline 5 & Caracterización & $\begin{array}{l}\text { Referidas a la definición y establecimiento de } \\
\text { procesos, mecanismos, fenómenos, hechos se- } \\
\text { gún características comunes. }\end{array}$ & 9 \\
\hline 6 & $\begin{array}{l}\text { Diseño, construcción y evaluación } \\
\text { de herramientas digitales }\end{array}$ & & 9 \\
\hline 7 & $\begin{array}{l}\text { Diseño, construcción y evaluación } \\
\text { de modelos conceptuales }\end{array}$ & & 5 \\
\hline 8 & Recomendaciones & $\begin{array}{l}\text { Entendidas como las sugerencias y/u opiniones } \\
\text { derivadas de los análisis y deber ser o estado } \\
\text { del arte según el problema estudiado. }\end{array}$ & 4 \\
\hline 9 & $\begin{array}{l}\text { Diseño, construcción y evaluación de } \\
\text { plataforma física experimental }\end{array}$ & & 2 \\
\hline 10 & $\begin{array}{l}\text { Diseño, construcción y evaluación de } \\
\text { metodologías para el procesamiento, } \\
\text { registro, publicación y consulta } \\
\text { de información digital - telemática }\end{array}$ & & 1 \\
\hline 11 & $\begin{array}{l}\text { Diseño, construcción y evaluación } \\
\text { de propuesta arquitectónica }\end{array}$ & & 1 \\
\hline \multirow[t]{2}{*}{12} & $\begin{array}{l}\text { Diseño, construcción y evaluación } \\
\text { de criterios y acciones }\end{array}$ & & 1 \\
\hline & TOTAL & & 152 \\
\hline
\end{tabular}

Fuente: Protocolos e informes de investigación CONDES y estimación propia (Bravo, 2000). 
tos). Le siguen los "procesos de evaluación y análisis" con el $22 \%$ y los "modelos y métodos de evaluación y análisis" con el $18 \%$. Estos 95 productos, representan una tercera parte del total de los productos generados por dichos proyectos.

\subsection{Productos derivados o conexos generados}

Como productos derivados o conexos, se obtiene que el $40 \%$ de los mismos son publicaciones (libros, artículos, memorias en eventos). También es significativa la asistencia a eventos (25\%), los contactos institucionales (17\%) y las actividades educativas (14\%), entre otras.

\subsection{Relación montos globales ejecutados y productos generados}

La doble relación porcentual entre montos globales ejecutados por cada uno de los proyectos de investigación IFADCONDES y el monto total ejecutado en los 23 proyectos de investigación y entre los productos generados (directos+indirectos) por cada proyecto de investigación respecto al total de productos generados en el conjunto de proyectos, se muestra en el Gráfico 10. En este gráfico se observa que la mayoría de los proyectos de investigación aprobados entre los años 1987 y 1992 (9 de 10 proyectos de investigación), han ejecutado mucho más

\section{Gráfico 10}

Relación porcentual entre el monto global ejecutado por cada proyecto de investigación IFAD-CONDES y el total ejecutado en los 26 proyectos y entre los productos generados por cada proyecto y el total de productos generados en los 26 proyectos

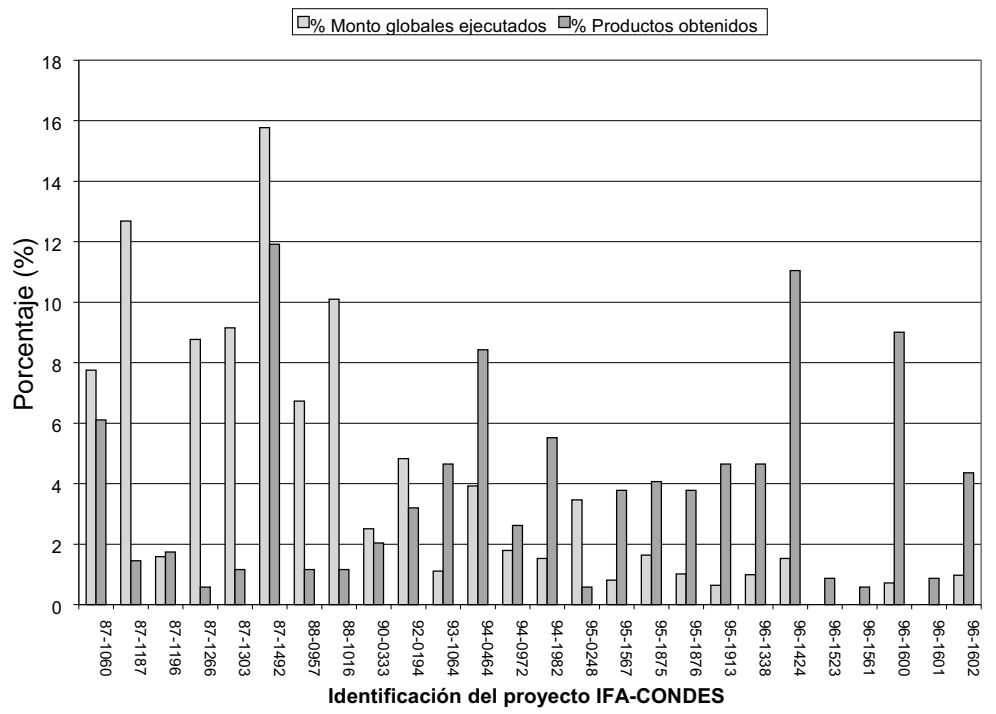

Fuente: Estimación propia (Bravo, 2000). 
dinero que lo generado como productos de la investigación. Mientras que, en un total de 13 proyectos aprobados entre 1993 y 1996, 12 proyectos han generado más cantidad de productos en relación con el monto ejecutado. Esto último, pudiera asociarse con las políticas de producción y divulgación asumidas por el CONDES durante los últimos años de este estudio.

El establecimiento de cuáles proyectos fueron más productivos que otros durante el período de estudio y la metodología para la estimación de productividad, son tratados en otro artículo (Bravo, 2001).

\section{Conclusiones}

La caracterización científica de una organización de investigación universitaria no se limita exclusivamente a la producción obtenida a través de los proyectos de investigación financiados por los $\mathrm{CDCHT}$, sino que abarca un conjunto mayor de productos derivados de todas las actividades científicas y gerenciales realizadas en el seno de la organización. Sin embargo, pese a las limitaciones de información presentes en estas organizaciones y al alcance de la investigación, esta metodología permite sistematizar y formalizar la recolección y análisis de algunos procesos y resultados de la actividad científica aplicables a estas organizaciones universitarias.

Es importante destacar que la información recopilada, procesada y analizada forman parte de la construcción de la memoria tecnológica de la unidad de investigación, la cual es vital para el apren- dizaje tecnológico y para el desarrollo de innovaciones tecnológicas. Asimismo, los resultados obtenidos sobre la producción científica, se constituyen en una información esencial para la toma de decisiones sobre este respecto en la planificación y gerencia de las unidades de I y D, siendo un tema importante dentro de la Planificación y Gerencia de la Ciencia y Tecnología y la gerencia universitaria.

En referencia al caso de estudio, se establecieron una serie de correlaciones entre los insumos y productos generados en los proyectos IFAD-CONDES para determinar la existencia o no de algún tipo de correspondencia. En este sentido, y sobre la base de la totalidad de los proyectos IFAD-CONDES se obtiene las siguientes conclusiones:

El monto global ejecutado y la duración del proyecto tienen poca influencia en la cantidad de productos generados.

El tiempo global de dedicación del personal de investigación tiene una fuerte relación con la duración del proyecto IFAD-CONDES y, por lo tanto, con el monto global ejecutado del proyecto de investigación IFADCONDES. Es decir, que con tiempos globales mayores en la dedicación del personal de investigación, se obtuvieron mayores montos globales y a mayor duración de los proyectos, mayores fueron los montos globales ejecutados. Esta fuerte correlación se debió básicamente al monto ejecutado específicamente por el personal docente y de investigación, que también dependió a su vez del tiempo de ejecución del proyecto. 
No se encontró correlación entre la duración del proyecto y el tiempo de adquisición de los equipos, el tiempo de dedicación semanal del personal de LUZ y el tiempo de dedicación semanal del personal contratado, el monto global ejecutado y los tiempos de dedicación semanal del investigador responsable o del co-investigador, la dedicación del personal de LUZ en la universidad o su categoría, con respecto al tiempo de dedicación semanal al proyecto, la cantidad o número de productos generados por proyecto de investigación IFAD-CONDES y el tiempo de dedicación total o global del personal de investigación (personal de LUZ y contratado).

\section{Recomendaciones}

Las recomendaciones de la investigación para ejecutarse a corto y mediano plazo, atienden aspectos relacionados con la presentación y contenido de las memorias y cuentas de las diferentes dependencias universitarias, los contenidos de los informes finales y protocolos de investigación de los proyectos, el registro de las actividades desarrolladas en la unidad de investigación estudiada, las acciones gerenciales propuestas para la organización en función de los resultados obtenidos de este estudio, y nuevos estudios o futuras investigaciones a desarrollar en el seno de la institución. Para los efectos de este documento, solamente se hace mención de las acciones gerenciales propuestas para la unidad de investigación en función de los resultados obtenidos de este estudio y con el propósito de potenciar la actividad y producción científica. En este sentido, se recomienda lo siguiente:

Establecer prioridades de investigación en función de un número limitado de áreas problemas y sus líneas específicas de investigación.

Sistematizar el establecimiento de objetivos, políticas, estrategias y planes basándose en la gestión explícita de proyectos de investigación y desarrollo ( y $\mathrm{D}$ ), orientada hacia la generación de innovaciones y desarrollos tecnológicos. En este sentido, la institución debería supervisar y proteger la propiedad intelectual de todos los productos de investigación, estableciendo claramente los regímenes de co-participación intelectual (si la hubiere) del personal contratado y tesistas adscritos a los proyectos frente al derecho de la universidad como propietaria de toda su producción académica. Así como también, gestionar modalidades de registro sistemático de propiedad intelectual y de patentes. Dicha gestión, así como otras de carácter administrativo (trámites para financiamiento o co-financiamiento de proyectos, entre otros) podrían realizarse a través de la creación de una oficina de gestión en la Facultad con personal técnico especializado. De esta manera, se reducirían los tiempos y los costos implicados en la dedicación a estas labores del personal docente y de investigación de alto nivel o formación dentro de la ejecución de los proyectos de investigación.

Establecer estrategias explícitas de promoción y vinculación institucional a través de tales medios que pudieran 
convergir en contratos de servicios tecnológicos y convenios de cooperación interinstitucional. Con ello, no solo se lograría potenciar la disponibilidad de recursos de inversión en investigación sino incluso, los modos de comercialización y aplicación del conocimiento generado.

Definir estrategias para el co-financiamiento de los proyectos de investigación con otras instituciones extrauniversitarias y coordinadas con otras instancias de la universidad.

Establecer un plan orientado hacia el refuerzo de los recursos humanos y tecnológicos con los que podrá contar el IFAD para confrontar tales situaciones en los próximos años dentro de un plan integral de gestión de la $\mathrm{Fa}$ cultad de Arquitectura y Diseño. En este sentido, se requeriría de un plan de formación y actualización académica de alto nivel del personal adscrito al IFAD en dichas áreas de conocimiento bajo una óptica gerencial de planificación, monitoreo y manejo de proyectos de I y $\mathrm{D}$, así como de un plan de inversión de recursos tecnológicos para el establecimiento de laboratorios de experimentación y un sistema integrado de documentación institucional.

\section{Bibliografía Citada}

Azuaje, Julia de (1987). El Centro Vitícola del Estado Zulia y su aporte al desarrollo de la vitivinicultura tropical en Venezuela, Tesis de Postgrado de la Maestría Planificación y Gerencia de Ciencia y Tecnología, Venezuela. Facultad Experimental de Ciencias. La Universidad del Zulia.
Banco Central de Venezuela - BCV (2000), Indice General de Precios al Consumidor (IPC). Area Metropolitana de Caracas, www.bcv.org.ve, diciembre 1999.

Bravo, Gaudy (1996). Propuesta de Gerencia de I y D. Instituto de Investigaciones de LUZ. Trabajo presentado en la Maestría de Planificación y Gerencia de Ciencia y Tecnología, Venezuela, La Universidad del Zulia, Facultad Experimental de Ciencias.

Bravo, Gaudy (2000). Caracterización de la producción científica de una unidad de investigación universitaria. Caso de Estudio: Instituto de Investigaciones (IFAD) de la Universidad del Zulia. Trabajo Especial de Grado. Maestría de Planificación y Gerencia de Ciencia y Tecnología, Venezuela, La Universidad del Zulia, Facultad Experimental de Ciencias.

Bravo, Gaudy (2001), Propuesta para estimar la productividad científica de proyectos de investigación en unidades de investigación universitaria, ESPACIOS Revista venezolana de gestión tecnológica, Volumen. 22, Número 2, Venezuela. CONICIT. pp. 61-72.

Castro, Arturo (1994), Estudio de mecanismos de vinculación del Instituto Universitario de Tecnología de Cabimas con el sector industrial de la Costa Oriental del Lago, Tesis de Postgrado de la Maestría Planificación y Gerencia de Ciencia y Tecnología, Venezuela. Facultad Experimental de Ciencias, La Universidad del Zulia.

Centro de Investigaciones Urbanas y Regionales - CIUR (1995), 10mo. Aniversario, Revista CIUR 16, Editorial Universitaria. Venezuela.

Consejo de Desarrollo Científico y Humanístico-CONDES (1986-1996), Memorias y Cuentas CONDES. La Universidad del Zulia, Vice-Rectorado Académico. 
Consejo Nacional de Investigaciones Científicas y Tecnológicas de Venezuela CONICIT (1995), Indicadores de la Capacidad de la Investigación en Ciencia y Tecnología, Caracas, Venezuela.

De Vries, Wietse (1998), Indicadores para la investigación: Usos y Abusos. México, Benemérita Universidad Autónoma de Puebla, vriesnav@gemtel. com.mx, julio 1999.

Ferrer de Esis, Brígida (1997), Apuntes sobre la Gerencia de Proyectos de I y D. Maestría en Planificación y Gerencia de Ciencia y Tecnología, Venezuela, La Universidad del Zulia, Facultad Experimental de Ciencias.

Figueroa, Marta (1995), Propuesta de gerencia para un Instituto de I y D. Estudio de Caso: Instituto de Investigaciones Petroleras de la Universidad del Zulia, Tesis de Postgrado de la Maestría Planificación y Gerencia de Ciencia y Tecnología. Venezuela. Facultad Experimental de Ciencias, La Universidad del Zulia.

González, Maigualida (1995), Una estructura organizativa para la vinculación educación - industria. El caso del Departamento de Investigación del Instituto Universitario deTecnología de Cabimas, Tesis de Postgrado de la Maestría Planificación y Gerencia de Ciencia y Tecnología. Venezuela. Facultad Experimental de Ciencias, La Universidad del Zulia.

Kauffman, James y Asociados (1998), Contadores públicos, Tasas de cambio mensual del Bolívar en relación con el dólar estadounidense. Maracaibo, Venezuela.
Licea de Arenas, Judith (1993), Indicadores de la actividad científica, Revista Ciencias de la Información, Volumen 24, Número 1, Cuba. PROINFO/SOCIT.

Licha, Isabel (1993), Capacidad de Gestión de Centros de Investigación en Venezuela, Consejo de Desarrollo Científico y Humanístico. Caracas, Venezuela. Universidad Central de Venezuela.

López, Romualdo (1998), Indicadores para evaluar áreas de investigación, Azcapotzalco, México, Universidad Autónoma Metropolitana, www.azc.uam. mx, junio 1999.

Paredes, Leopoldo (1993), Planificación, Control y Evaluación de Proyectos de Investigación y Desarrollo. Manual, Venezuela, La Universidad del Zulia, Ediciones Astro Data S.A.

Peña, Jesús y Barrios, Manuel (1994), Diseño Conceptual y Metodológico (versión resumida). Programa de Evaluación de Centros de Investigación y desarrollo (PECID), Caracas, División de Estudios Especiales, Dirección de Política y Planificación, CONICIT.

La Universidad del Zulia - LUZ (1978), Reglamento del Instituto de Investigaciones de Arquitectura y Sistemas Ambientales - ISA, Consejo Universitario, Maracaibo, Venezuela.

U.S. Department of Labor Bureau of Labor Statistics (2000), Consumer Price Index. All Urban Consumers (CPI-U), U.S. City average, Washington, D.C. 20212, ftp://rtp.bls.gov/pub/special. Requests/cpi/cpiai.txt, diciembre 1999. 